\title{
Sagittal Balance of the Spine in Daily Practice:A Survey among Spine Surgeons in the Netherlands
}

\begin{abstract}
Purpose: To provide an overview of the influence of the sagittal balance concept on diagnosis and decision making among spine surgeons in the Netherlands.

Methods: A survey was conducted amongst members of the Dutch Spine Society, which includes both neurosurgeons and orthopedic surgeons. The survey posed questions concerning the application of the sagittal balance concept in clinical practice, diagnostic work-up, and its influence on decision-making and surgical outcome.
\end{abstract}

Results: Among spine surgeons in the Netherlands, there is widespread support for the theory of the sagittal balance of the spine in clinical practice. Neurosurgeons apply it clinically less often and perform less diagnostics to determine the sagittal balance than orthopedic surgeons. When applied, for most respondents the sagittal balance concept influence clinical decision-making, but fewer think that it improves patients' outcomes.

Conclusion: In the Netherlands, most spine surgeons are familiar with the theory of the sagittal balance, but its clinical application thus far is limited, especially amongst neurosurgeons.

\author{
Volume 3 Issue 6 - 2015
}

\author{
Ochtman AEA,' Goulin Lippi Fernandes E,' \\ Willems PC, ${ }^{2}$ Öner FC, ${ }^{3}$ van Gaalen SM' \\ 'Department of Orthopedics, Clinical Orthopedic Research \\ Center midden-Nederland (CORC-mN), Netherlands \\ ${ }^{2}$ Department of Orthopedics, Maastricht University Medical \\ Center, Netherlands \\ ${ }^{3}$ Department of Orthopedics, University Medical Center \\ Utrecht, Netherlands
}

\begin{abstract}
Correspondence: Ochtman AEA, CORC-mN, Department of Orthopedics, Diakonessenhuis Utrecht/Zeist, PO Box 80250,3508 TG, Utrecht, Netherlands, Tel +3I 88250 5087, Email lochtman@diakhuis.nl
\end{abstract}

Received: December 0I, 2015 | Published: December 23, 2015

Keywords: Sagittal balance; Spine; Survey; Dutch spine society

\section{Introduction}

Low back pain (LBP) is globally an important health problem as it is the number one cause of disability in modern society with huge subsequent indirect costs due to lost economic productivity. ${ }^{1}$ LBP has a life-time prevalence in the Netherlands as high as $60-90 \%$ in the general (/family) practice population. ${ }^{2}$ and up to $84 \%$ worldwide. ${ }^{3}$ Degenerative spinal disorders are held responsible for most of the symptoms. ${ }^{4}$ With ageing, degenerative processes cause a decrease of lumbar lordosis, thereby altering the balance of the spinal column in the sagittal plane. ${ }^{5,6}$ Retroversion of the pelvis, hyperextension of the hip joints and flexion of the knees are physiological compensatory mechanisms in order to maintain the body in an upright posture. ${ }^{7}$ This mechanism gradually loses its capacity to compensate because of further decrease of the lumbar lordosis and/or increase of the thoracic kyphosis, particularly due to loss of intervertebral disc heights. ${ }^{8}$

These compensatory mechanisms increasingly cause low back pain, because of overstress and facet constraints [8]. Conversely, the sagittal balance can be negatively influenced by spine surgery, such as vertebral fusion, as the vertebrae may have been fused with a decrease of the spinal curvature, leading to forward inclination of the trunk. ${ }^{9-13}$ An elevated pelvic tilt, reduced lumbar lordosis and sagittal imbalance are generally associated with poorer functional outcomes after surgery. ${ }^{14}$

Spine surgeons increasingly recognize the importance of maintaining and improving the sagittal balance for a better clinical outcome. Accordingly, the influence of sagittal balance parameters on decision-making in clinical practice is increasing in the Netherlands. This study was aimed to provide an overview of the current influence of the sagittal balance of the spine on the decision-making of spine surgeons in the Netherlands.

\section{Material and methods}

A cross-sectional survey containing both multiple-choice and open questions was produced under supervision of 3 spine surgeons (PW, SvG and CÖ). All members of the Dutch Spine Society (DSS), the professional organization of all spine surgeons in the country, both orthopedic surgeons and neurosurgeons, were invited to participate in the survey on paper on November 7, 2014 at the annual meeting of the society. Since not all members of the DSS were present at this meeting, the remaining members were approached by e-mail on November 15 , 2014. This e-mail contained a unique link to an electronic version of the same survey. A final call for participation in the survey was made in the newsletter of the DSS, one month after the annual meeting. The surveys were completed anonymously.

\section{Data analysis}

The data from the completed surveys was entered into Microsoft Excel (Microsoft Corp., Redmond, Washington, USA). Statistical analyses were performed with Statistical Package for the Social Sciences software (SPSS 22.0, SPSS Inc., Chicago, Illinois, USA. ${ }^{15}$ ). The Fisher's exact test was employed to calculate the statistical significance of contingency tables.

\section{Results}

At the time of the survey, the DSS consisted of 178 members: 107 orthopedic surgeons $(60 \%), 61$ neurosurgeons $(34 \%)$ and 10 non-surgeon members $(6 \%)$. Of those, 81 members declared not to perform spinal fusion operations and were therefore excluded from the survey. Of the remaining 87 orthopedic and neurosurgeons, 60 $(68.9 \%)$ completed the survey (43 orthopedic surgeons (72\%), 17 neurosurgeons $(28 \%))$. The main characteristics of the participants are displayed in Table 1. Both neurosurgeons and orthopedic surgeons have a comparable clinical experience and consider the theory of sagittal balance important (98\% of orthopedic surgeons, $88 \%$ of neurosurgeons, $\mathrm{p}=0.191)$. However, orthopedic surgeons apply the theory of sagittal balance in clinical practice significantly more often $(83.7 \%$ vs. $29.4 \%, \mathrm{p}<0.0001)$. 
As displayed in Table 2, most specialists who consider the sagittal balance to be important also apply it in clinical practice. However, 10 of the 15 neurosurgeons who find the conceptual framework relevant, do not apply it in clinical practice, versus 7 of the 42 orthopedic surgeons who do find it relevant. (Remarkably, one neurosurgeon applies the sagittal balance concept in daily practice, but does not find the theory relevant).

Surgeons, who use the sagittal balance in clinical practice, perform radiological investigations of the spine and the physical examination of the hips significantly more often (Table 2 ) (both $\mathrm{p}<0.001$ ). Also, orthopedic surgeons perform radiological investigations by means of radiograms and physical examination of the hips significantly more often than neurosurgeons $(\mathrm{p}<0.001$ and $\mathrm{p}<0.0001$, respectively). Most surgeons (92\%) use the sagittal balance as a factor for decision making, and a small minority of those (42\%) also thinks that patient outcomes will improve as a result of that (Table 3). The sagittal balance is considered decisive for the choice of surgical treatment for $89 \%$ of surgeons. The amount of experience using the sagittal balance concept in clinical practice does not significantly influence its role in decision-making.

Table I Baseline characteristics.

\begin{tabular}{|c|c|c|c|}
\hline Specialism & Orthopedic Surgery & Neurosurgery & P-Value \\
\hline Group Size & $43 / 60(72 \%)$ & $17 / 60(28 \%)$ & - \\
\hline Experience (Years) & 12.58 (SD 10.85) & I3.73 (SD 9.1 I) & 0.72 \\
\hline Q.I:Thinks Theory of Sagittal Balance is Clinically Relevant & $42 / 43(98 \%)$ & $15 / 17(88 \%)$ & 0.191 \\
\hline Q.4:Applies Sagittal Balance in Clinical Practice & $36 / 43(84 \%)$ & $5 / 17(29 \%)$ & $<0.0001$ \\
\hline Q.2: Orders X-Ray of Spine pre-op & $40 / 43(93 \%)$ & $8 / 17(47 \%)$ & $<0.001$ \\
\hline Q.3: Performs pre-op hip Examination & $42 / 43(98 \%)$ & $6 / 17(35 \%)$ & $<0.0001$ \\
\hline
\end{tabular}

Table 2 Theory of sagittal balance versus its clinical application

\begin{tabular}{|c|c|c|c|c|}
\hline & & \multicolumn{3}{|c|}{ Q.4:Applies Sagittal Balance In Clinical Practice P-Value } \\
\hline & & Yes & No & \\
\hline \multirow[t]{2}{*}{ Q.I } & $\begin{array}{l}\text { "Theory of Sagittal Balance is } \\
\text { Clinically Relevant" }\end{array}$ & 40 & 17 & \multirow[t]{2}{*}{0.233} \\
\hline & "Sagittal Balance is a Hype" & I & 2 & \\
\hline \multirow{2}{*}{$\begin{array}{l}\text { Q. 2:Always Orders X-ray of Spine } \\
\text { pre-op }\end{array}$} & Yes & 38 & 10 & \multirow{2}{*}{$<0.001$} \\
\hline & No & 3 & 9 & \\
\hline \multirow{2}{*}{$\begin{array}{l}\text { Q.3:Always Performs Hip Examination } \\
\text { pre-op }\end{array}$} & Yes & 39 & 9 & \multirow{2}{*}{$<0.0001$} \\
\hline & No & 2 & 10 & \\
\hline
\end{tabular}

Table 3 Consequences of applying the sagittal balance in clinical practice

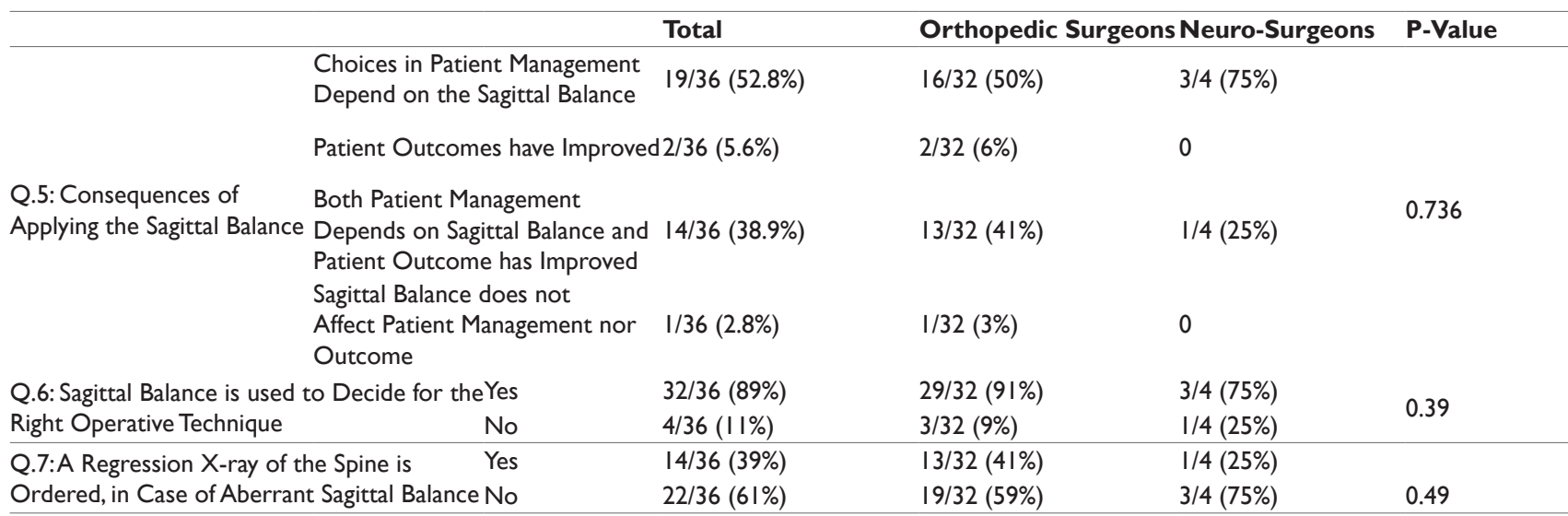

\section{Discussion}

This study was the first to investigate the current state of affairs regarding the acceptance and use of sagittal balance concept in spine surgery, by performing a survey amongst Dutch neurosurgeons and orthopedic spine surgeons. We found that an overwhelming majority $(95 \%)$ of spine surgeons in the Netherlands, both neurosurgeons and orthopedic surgeons, consider the theory about the sagittal balance enrichment for the clinical practice. However, a far lower number of neurosurgeons (27\%) apply the theory of the sagittal balance in their clinical practice, compared to orthopedic surgeons $(84 \%)$. Accordingly, neurosurgeons significantly perform less preoperative investigations that pertain to the sagittal balance parameters, namely the hip examination and conventional full-spine radiograms. Most neurosurgeons and orthopedic surgeons, who use the sagittal balance concept in daily practice, use it to guide their decision-making. Surprisingly, a much smaller percentage of those perceived that the introduction of the sagittal balance concept in clinical practice will lead to an improvement of patient outcomes.

The main challenge that was encountered in this study was the gap between widespread support for the theory of sagittal balance concept, and its clinical application, especially amongst neurosurgeons. There are no prior surveys concerning the practice of the sagittal balance concept for comparison, and there are only a small number of surveys that compared neurosurgeons and orthopedic surgeons. Two surveys showed that orthopedic surgeons and neurosurgeons significantly differ in decision-making in spine surgery. ${ }^{16,17}$ 
One survey, taken in Korea, showed significant differences regarding the management of various spinal disorders. ${ }^{17}$ Significantly more orthopedic surgeons were in favor of aggressive discectomy versus fragmentectomy in case of herniated disc surgery. Orthopedic surgeons were also significantly more often in favor of surgical intervention for traumatic spinal cord injuries. Lastly, more neurosurgeons were in favor of non-instrumented fusions, although this difference was not significant. The other survey was amongst spine surgeons in the USA and showed that orthopedic surgeons were significantly more likely to use instrumentation for degenerative lumbar spinal disorders. ${ }^{16}$ Training could be the foundation of these differences in management and decision-making, and it could also have its effect on differences in clinical application of the sagittal balance between neurosurgeons and orthopedic surgeons. A recent European study assessed the theoretical and practical skills of neurosurgeons and orthopedic surgeons who had or had not participated in a 1-year spine fellowship. ${ }^{18}$

There was no difference between orthopedic surgeons and neurosurgeons with regard to self- reported knowledge of the theory or practical skills. The spine fellowship did not significantly increase theoretical knowledge for most areas, except for spinal deformity This underlines that young European neurosurgeons and orthopedic surgeons have a thorough theoretical knowledge of spinal disorders, although knowledge about the sagittal balance was not specifically addressed. This might reflect the widespread support for the theory of the sagittal balance in our study. The practical skills competency did show significant differences between surgeons in favor of those who had participated in the fellowship. This could mean that more practical and hands-on training could lead to a more widespread clinical application of the sagittal balance concept in the Netherlands.

Most spine surgeons who apply the sagittal balance concept in clinical practice use it to guide their decision-making. Interestingly, a much smaller percentage thinks consideration of the sagittal balance has led to better surgical outcomes. Available literature regarding this topic is limited. No trials have been published today that compared surgical outcomes between patients who received correction of the sagittal balance versus no correction. Several authors have studied the relationship between radiologic and clinical outcome parameters and found that a correct sagittal balance leads to better clinical outcome. ${ }^{14,19,20}$ However, studies with higher levels of evidence would shed more light on the magnitude or absence of benefits of applying the sagittal balance. Compensation mechanisms, such as retroversion of the pelvis and hyperextension of the hip joints, have been studied and are found to have a significant influence on the sagittal alignment. ${ }^{21,22}$

This suggests that physical examination of the hips to diagnose hip osteoarthritis, which prevents the hips from hyperextension and the pelvic to tilt backwards, cannot be ignored when applying the sagittal balance concept in daily practice and is essential to consider for every spine surgeon when a patient with LBP is evaluated. We invited every practicing surgeon of the nationwide Dutch Spine Society to participate in this review, thereby guaranteeing that all spine surgeons of the country had been invited. Furthermore, this survey had an excellent response rate $(69 \%)$ compared to similar surveys amongst neurosurgeons and orthopedic surgeons. ${ }^{18,23,24}$ including a recent Dutch survey with members of the DSS. ${ }^{25}$

Moreover, since the questions in the survey had been tested by 3 spine surgeons and an epidemiologist prior to presentation to all spine surgeons, we were encouraged to assume that the questions were unambiguous and syntactically correct. This survey covered the most relevant aspects of spine surgery in relation to the sagittal balance: the opinion about the theoretical value, its implementation in clinical practice, the frequency of preoperative diagnostics pertaining to the sagittal balance, the effect of the sagittal balance on decision-making and the perceived surgical outcome. Hence, we were able to give a complete overview of the current state of the sagittal balance in spine surgery in the Netherlands. Additionally, by securing anonymity and by approaching all spinal surgeons regardless of experience, region, or training, we prevented bias.

This survey was made concise in order to discourage as few surgeons as possible from participating, but this also led to a number of shortcomings. We did not query the number of spine operations that each surgeon performs, and whether there is a subset of patients for whom the sagittal balance is not taken into account. There might be differences amongst orthopedic surgeons and neurosurgeons in this respect, which could have been explanatory with regard to the preoperative diagnostics pertaining to the sagittal balance. Neither was the distribution of indications for spine surgery nor the different spinal operative techniques to be used by the respondents in clinical practice investigated, which might also have explained differences between neurosurgeons and orthopedic surgeons. Additionally, although this survey questioned the experience of the surgeons, it was not taken into account how large the subset was of surgeons who had undergone a spine fellowship or specific sagittal balance training. Knowledge of the differences in training background could have elucidated the differences between orthopedic surgeons and neurosurgeons.

\section{Conclusion}

In the Netherlands, the theory of the sagittal balance has widespread support, but it is not yet applied clinically by the majority of orthopedic surgeons and neurosurgeons. We expect the number of surgeons using the sagittal balance concept to increase with more training and continued presentation of evidence in its favor.

\section{Ackowledgments}

None.

\section{Conflicts of interst}

None.

\section{References}

1. Lim SS, Vos T, Flaxman AD et al. A comparative risk assessment of burden of disease and injury attributable to 67 risk factors and risk factor clusters in 21 regions, 1990-2010: a systematic analysis for the Global Burden of Disease Study 2010. Lancet. 2012:380(9859):2224-2260.

2. van Tulder MW, Koes BW, Bouter LM A cost-of-illness study of back pain in The Netherlands. Pain. 1995:62(2):233-240.

3. Walker BF prevalence of low back pain: a systematic review of the literature from 1966 to 1998. J Spinal Disord. 2000:13(3):205-217.

4. Adams MA, Dolan $\mathrm{P}$ Spine biomechanics. $J$ Biomech. 2005:38(10):1972-1983.

5. Gelb DE, Lenke LG, Bridwell KH et al. An analysis of sagittal spinal alignment in 100 asymptomatic middle and older aged volunteers. Spine (Phila Pa 1976). 1995:20(12):1351-1358.

6. Korovessis PG, Stamatakis MV, Baikousis AG Reciprocal angulation of vertebral bodies in the sagittal plane in an asymptomatic Greek population. Spine (Phila Pa 1976). 1998:23(6):700-704.

7. Le Huec JC, Aunoble S, Philippe L et al. Pelvic parameters: origin and significance. Eur Spine J. 2011:20(Suppl 5): 564-571.

8. Le Huec JC, Charosky S, Barrey C et al. Sagittal imbalance cascade for simple degenerative spine and consequences: algorithm of decision for appropriate treatment. Eur Spine J. 2011:20(Suppl 5):699-703. 
9. Le Huec JC, Faundez A, Dominguez D et al. Evidence showing the relationship between sagittal balance and clinical outcomes in surgical treatment of degenerative spinal diseases: a literature review. Int Orthop. 2015:39(1):87-95.

10. Gödde S, Fritsch E, Dienst M et al. Influence of cage geometry on sagittal alignment in instrumented posterior lumbar interbody fusion. Spine (Phila Pa 1976). 2003:28(15):1693-1699.

11. Goldstein JA, Macenski MJ, Griffith SL et al.Lumbar sagittal alignment after fusion with a threaded interbody cage. Spine (Phila Pa 1976). 2001:26(10):1137-1142.

12. Stephens GC, Yoo JU, Wilbur G Comparison of lumbar sagittal alignment produced by different operative positions. Spine (Phila Pa 1976). 1996:21(15):1802-1806.

13. Tribus CB, Belanger TA, Zdeblick TA The effect of operative position and short-segment fusion on maintenance of sagittal alignment of the lumbar spine. Spine (Phila Pa 1976). 1999:24(1):58-61.

14. Mehta VA, Amin A, Omeis I et al. Implications of spinopelvic alignment for the spine surgeon. Neurosurgery. 2012:70(3):707-721.

15. IBM IBM SPSS Statistics for Windows. IBM Corp, Armonk, USA. 2013

16. Irwin ZN, Hilibrand A, Gustavel M et al. Variation in surgical decision making for degenerative spinal disorders. Part I: lumbar spine. Spine (Phila Pa 1976). 2005:30(19):2208-2213.

17. Hussain M, Nasir S, Moed A et al. Variations in Practice Patterns among Neurosurgeons and Orthopaedic Surgeons in the Management of Spinal Disorders. Asian Spine J. 2011:5(4):208-212.
18. Konczalik W, Elsayed S, Boszczyk B Experience of a fellowship in spinal surgery: a quantitative analysis. Eur Spine J. 2014:23(Suppl 1): S40-S54.

19. Schwab FJ, Blondel B, Bess S et al. Radiographical spinopelvic parameters and disability in the setting of adult spinal deformity: a prospective multicenter analysis. Spine (Phila Pa 1976). 2013:38(13): E803-E812.

20. Glassman SD, Bridwell K, Dimar JR et al. The impact of positive sagittal balance in adult spinal deformity. Spine (Phila Pa 1976). 2005:30(18):2024-2029

21. Weng WJ, Wang WJ, Wu MD et al. Characteristics of sagittal spinepelvis-leg alignment in patients with severe hip osteoarthritis. Eur Spine J. 2015:24(6):1228-1236.

22. Lafage V, Schwab F, Patel A et al. Pelvic tilt and truncal inclination: two key radiographic parameters in the setting of adults with spinal deformity. Spine (Phila Pa 1976). 2009:34(17):E599-E606.

23. Mesfin A, Canham C, Okafor L Prevention Training of Wrong-Site Spine Surgery. J Surg Educ. 2015:72(4):680-684.

24. Uribe JS, Deukmedjian AR Visceral, vascular, and wound complications following over 13,000 lateral interbody fusions: a survey study and literature review. Eur Spine J. 2015:24(Suppl 3):386-396.

25. Overdevest GM, Moojen WA, Arts MP et al. Management of lumbar spinal stenosis: a survey among Dutch spine surgeons. Acta Neurochir (Wien). 2014:156(11):2139-2145. 